\title{
Utility of the Gel Immersion Method for Treating Massive Colonic Diverticular Bleeding
}

\author{
Kazuki Yamamoto, Yasutoshi Shiratori and Takashi lkeya \\ Division of Gastroenterology, St. Luke's International Hospital, Tokyo, Japan
}

Background/Aims: In Asia, right-sided diverticular bleeding is more common than that of the left side. It often causes massive bleeding and difficulties in identifying the stigmata of recent hemorrhage (SRH) of colonic diverticular bleeding (CDB). This case series demonstrates the efficacy of the gel immersion method using OS-1 Jelly (Otsuka Pharmaceuticals Factory, Tokushima, Japan) in patients with $\mathrm{CDB}$.

Methods: This retrospective case series analyzed data of patients with CDB who underwent the gel immersion method from April 2016 to February 2020 at St. Luke's International Hospital, Japan. All patients diagnosed with CDB who underwent the gel immersion method were included. We collected data on the site of bleeding, identification of SRH, and efficacy of the method from the electronic medical records.

Results: A total of 9 patients (including 7 with right-sided CDB) underwent gel immersion method and were included in this study. SRH were successfully found in $66.7 \%$ (6/9) of patients. Moreover, effective hemostasis was achieved in $85.7 \%$ (6/7) of patients with right-sided CDB. There were no adverse events.

Conclusions: The gel immersion method was found to be effective, especially for massive right-sided CDB. Clin Endosc 2021;54:256260

Key Words: Diverticular diseases; Diverticulum; Gastrointestinal hemorrhage

\section{INTRODUCTION}

Lower gastrointestinal bleeding (LGIB) accounts for approximately $20 \%$ of all cases of gastrointestinal (GI) bleeding. ${ }^{1}$ Colonic diverticular bleeding $(\mathrm{CDB})$ is its most common type in adults; accounting for $30 \%-65 \%$ of LGIB. ${ }^{2,3}$ With aging, the prevalence is increasing worldwide ${ }^{4-6}$; and so are the number of emergency visits and admission rates, making $\mathrm{CDB}$ a significant global healthcare concern.?

Right-sided diverticular bleeding is more common in Asia,

Received: March 30, 2020 Revised: May 9, 2020

Accepted: June 9, 2020

Correspondence: Takashi Ikeya

Division of Gastroenterology, St. Luke's International Hospital, 9-1 Akashi-cho, Chuo-ku, Tokyo 104-8560, Japan

Tel: +81-3-3541-5151, Fax: +81-3-3541-5151, E-mail: takaike@luke.ac.jp

ORCID: https://orcid.org/0000-0003-4838-2207

(c) This is an Open Access article distributed under the terms of the Creative Commons Attribution Non-Commercial License (http://creativecommons.org/ licenses/by-nc/3.0) which permits unrestricted non-commercial use, distribution, and reproduction in any medium, provided the original work is properly cited. and it often causes massive bleeding with a potential risk of re-bleeding and complications. ${ }^{8-10}$ In Japan, $68 \%-76 \%$ of confirmed diverticular bleeding cases were right-sided. ${ }^{11,12}$ In approximately $75 \%$ of patients, CDB stops spontaneously; while bleeding persists in the remaining $25 \% .{ }^{13,14}$ However, the identification rates of the stigmata of recent hemorrhage $(\mathrm{SRH})$ defined as active bleeding $(\mathrm{AB})$, non-bleeding visible vessels (NBVVs), and adherent clot (AC), ${ }^{15}$ remain relatively low, ${ }^{16}$ and this is mainly due to two reasons. First, since the diverticular bleeding is intermittent, spontaneous hemostasis is more likely to occur, especially in left-sided diverticular bleeding, where the bleeding is relatively less. Second, is the inability to obtain a clear visual field in the presence of massive bleeding that is particularly problematic in right-sided diverticular bleeding that is common in Asia. Detecting small NBVV and AC hidden under accumulated blood clots is very challenging. ${ }^{17}$ The SRH identification rates were reported to be $17 \%-42 \%,{ }^{11,12}$ and re-bleeding frequently occurred in approximately $50 \%$ of untreated SRH patients. ${ }^{18}$ Surgical intervention may be needed for patients with uncontrollable bleeding. ${ }^{19,20}$ 
Therefore, endoscopic hemostasis is recommended for a colonic diverticulum with SRH; leading from this, improvement of SRH detection rate is the key for CDB management. ${ }^{1,15,18}$

The use of a water-jet scope was introduced by Ishii et al. as a safe and effective endoscopic treatment for CDB in 2010. ${ }^{21}$ Later, Niikura et al. proved its efficacy by showing the use of a water-jet scope as an independent predictive factor of SRH in CDB. ${ }^{11}$ However, in patients with massive bleeding, the injected water rapidly mixes with fresh blood in the lumen, compromising a clear endoscopic view, and complicating the identification of the bleeding source. Yano et al. first reported on the safety and simplicity of the gel immersion method in 2016. ${ }^{22}$ They injected OS-1 Jelly (Otsuka Pharmaceuticals Factory, Tokushima, Japan), a famous oral rehydration clear gel, instead of water through the accessory channel for securing a clear visual field during endoscopic treatment of patients with GI bleeding. The gel immersion method was reported to be effective for 17 patients with several types of GI bleeding. They included 3 patients with right-sided CDB; however, the efficacy of OS-1 Jelly in detecting small NBVV and AC in these patients was unclear. ${ }^{22}$ The approach for right-sided diverticular bleeding is more difficult because of the massive bleeding encountered. Additionally, the intermittent nature of the diverticular bleeding, makes small NBVV and AC even more difficult to detect. ${ }^{17}$ In a previous study, OS-1 Jelly was shown to improve the detection rate of $\mathrm{AB}^{22}$; however, in our case series, we focus on the efficacy of the gel immersion method in CDB patients, especially in detecting NBVV and AC observed in patients with massive right-sided diverticular bleeding.

\section{MATERIALS AND METHODS}

\section{Data collection}

This retrospective case series analyzed the data of all consecutive patients with diverticular bleeding who underwent gel immersion method from April 2016 to February 2020 at St. Luke's International Hospital, in Tokyo, Japan. We collected data on patient age, sex, extravasation during computed tomography (CT) scan, the use of bowel preparation, the site of bleeding, the identification of SRH, and the efficacy of the method, from the electronic medical records. A contrast-enhanced CT scan was performed by a board-certificated radiologist, and SRH were confirmed by board-certified members of the Japanese Society of Gastrointestinal Endoscopy. SRH were classified as follows: AB, NBVV, and AC. The hospital ethics committee approved this study (approval number: 19-R210). Since this was a retrospective study, the need for written consent was waived by the IRB. However, those who refused to participate in this study, after checking the details on our noti- fication website were excluded.

\section{Urgent colonoscopy and gel immersion method}

Urgent colonoscopy was performed within 24 hours of admission, and bowel preparation with polyethylene glycol was performed before each procedure. However, for patients who were hemodynamically unstable and were receiving adequate intravenous fluid resuscitation or blood transfusion, bowel preparation was omitted. Contrast-enhanced CT was performed before the procedure for all patients who had agreed. PCF-Q260AZI, PCF-Q260JI, or PCF-H290I (Olympus, Tokyo, Japan) was mainly used to diagnose CDB. A transparent plastic cap (D-201-12704; Olympus Medical Systems) was attached to the endoscope to improve the detection rate. Butylscopolamine bromide or glucagon was administered at the time of testing, for suppressing GI peristalsis. A total of 6 expert endoscopists with an experience of over 1,000 colonoscopies, ${ }^{23}$ who were board-certified members of the Japanese Society of Gastrointestinal Endoscopy, performed all the endoscopies. Along with the experts, 4 trainees also performed the procedures under on-site expert supervision.

Although a water-jet scope is normally used, blood clots are so massive in some cases that securing a clear endoscopic view becomes very difficult. Although there were no clear criteria, we performed the gel immersion method if sufficient air supply, use of water jets, and repositioning were ineffective to obtain luminal vision. OS-1 Jelly was injected slowly using an irrigation accessory, the BioShield irrigator (US Endoscopy, Mentor, OH, USA). We stopped air insufflation and continued to inject the gel until a clear view was secured. Depending on the situation, we used an average of 2-3 packs of OS-1 jelly per CDB, with each pack containing $200 \mathrm{~mL}$ (Supplementary video 1) of jelly.

\section{RESULTS}

A total of 9 patients underwent gel immersion method and were included in this study. Table 1 depicts the characteristics of all patients, including age, sex, extravasation during CT scan, the use of bowel preparation, the site of bleeding, the identification of SRH, and the efficacy of the method. The mean age was 71.1 years, and the sample consisted of more men than women. Extravasation was positive in $44.4 \%$ (4/9) of patients, while $88.9 \%$ (8/9) underwent bowel preparation, and $77.8 \%$ (7/9) were diagnosed with right diverticular bleeding. The bleeding source identification and hemostatic treatment rate was $66.7 \%$ (6/9). Moreover, in terms of right-sided diverticular bleeding, effective hemostasis was obtained in $85.7 \%$ (6/7) of patients. Extravasation was observed in $42.9 \%$ (3/7) of 
Table 1. Characteristics of Nine Patients Treated with Gel Immersion Endoscopy

\begin{tabular}{cccccccc}
\hline No. & Age & Sex & Extravasation of CT scan & Bowel preparation & Site & SRH & Efficacy \\
\hline 1 & 92 & M & Positive & No & Right colon & AB & Effective \\
2 & 87 & F & Positive & Yes & Left colon & Negative & Not effective \\
3 & 65 & F & Negative & Yes & Right colon & NBVV & Effective \\
4 & 91 & M & Positive & Yes & Right colon & AB & Effective \\
\hline 5 & 64 & M & Negative & Yes & Right colon & Negative & Not effective \\
6 & 41 & M & Negative & Yes & Right colon & AC & Effective \\
\hline 7 & 86 & M & Positive & Yes & Right colon & AC, NBVV & Effective \\
8 & 60 & M & Not conducted & Yes & Left colon & Negative & Not effective \\
9 & 54 & M & Negative & Yes & Right colon & AB & Effective \\
\hline
\end{tabular}

$\mathrm{AB}$, active bleeding; $\mathrm{AC}$, adherent clot; CT, computed tomography; NBVV, non-bleeding visible vessel; SRH, stigmata of recent hemorrhage.
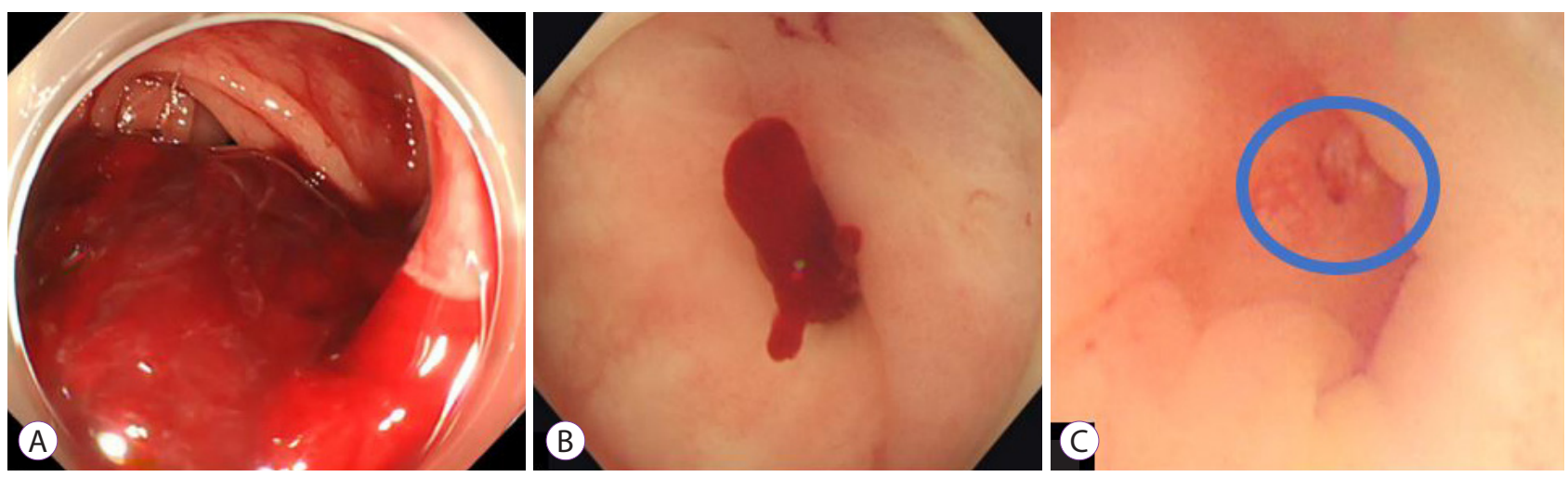

Fig. 1. (A) Colonoscopic view of massive bleeding in the right colon. (B) The gel immersion method successfully washed out the massive clots and revealed diverticula with adherent clot. (C) Peeling of the clot exposed a non-bleeding visible vessel.

patients with right-sided diverticular bleeding. In all patients (3/3) with extravasation from right-sided diverticular bleeding, effective hemostasis was obtained. Even when extravasation was absent, effective hemostasis was obtained in $75.0 \%$ (3/4) of patients with right-sided diverticular bleeding.

Fig. 1. shows a typical right-sided diverticular bleeding and the examination process by using OS-1 Jelly for identifying SRH of a diverticulum, in a patient with $\mathrm{CDB}$, hidden under massive blood clots. Fig. 1A. presents a colonoscopic view of the massive blood clots often found in patients with right-sided diverticular bleeding. A large number of clots had accumulated within the colon, interfering with a clear view and obstructing the identification of SRH. Fig. 1B. is a colonoscopic view after using OS-1 Jelly. Blood clots were successfully washed out, and viscosity of the gel prevented its rapid mixing with blood, contributing to improvement in the visual field that enabled the detection of an AC in the diverticulum. We used a spray catheter with a working length of $240 \mathrm{~cm}$ (PW1V1; Olympus, Tokyo, Japan) as a washing tube, gently peeling off the clot in the diverticulum. Fig. 1C. shows the exposed NBVV after gentle peeling of the AC.

\section{DISCUSSION}

$\mathrm{CDB}$ is known as the most common type of LGIB among adults, accounting for $30 \%-65 \%$ of LGIB. ${ }^{2,3}$ Its prevalence is increasing worldwide, ${ }^{4-6}$ yet the SRH identification rates remain low. ${ }^{16}$ There are mainly two reasons for the lower detection rates of SRH. Firstly, the diverticular bleeding is intermittent; thus, spontaneous hemostasis is more likely to occur, especially on the left side where the bleeding is relatively 
less. Secondly, it is difficult to obtain a clear visual field with massive bleeding that is particularly problematic in right-sided diverticular bleeding that is common in Asia. ${ }^{17}$ In terms of the predictors for identification of SRH in colonic diverticula, Niikura et al. reported urgent colonoscopy within 24 hours of onset of LGIB, use of a cap, use of a water-jet scope, and experienced colonoscopists as independent factors that may improve the detection rate of SRH. ${ }^{11}$ The timing of endoscopy is still debated, but in many cases, urgent endoscopy is recommended, and extravasation on contrast-enhanced CT improves the bleeding source identification. ${ }^{16}$ However, the positive rate of extravasation on CT is only $15.4 \%$, and thus, we cannot use CT for all patients. ${ }^{24}$

Diverticular bleeding occurs in 5\%-15\% of patients with diverticulosis and causes massive bleeding in one-third of patients. ${ }^{7}$ In patients with massive bleeding, fresh blood and massive clots obstruct a clear endoscopic view, making identification of SRH tremendously difficult. The use of a water-jet scope is useful for detecting the site of the bleeding source. ${ }^{11}$ Yet, as Yano et al. described in their study, when an area with a large amount of clots is washed with water, the injected water mixes instantaneously with blood, making it difficult to secure a clear visual field. ${ }^{22}$

In all of our 9 patients who underwent gel immersion method, the rate of detection of SRH on colonoscopy was markedly higher, especially in patients with right colon bleeding, and effective hemostasis was obtained in $85.7 \%$ (6/7) of patients. The rate of identification of $\mathrm{SRH}$ of a diverticulum in $\mathrm{CDB}$ that is extravasation-negative on CT is as low as $20 \%,{ }^{16}$ whereas our study showed a higher rate $(75.0 \%, 3 / 4)$ of effective hemostasis in patients with extravasation-negative right-sided diverticular bleeding. Although there were only two patients with left-sided diverticular bleeding, the gel immersion method did not increase the identification rates of SRH in left-sided CDB. One possible reason for this is that the left colon is a tortuous area where the scope is not stable, so the gel immersion method may not have been as effective as it was in the right colon, which has a wider lumen and is more prone to gel retention.

In general, NBVV and AC are said to be difficult to judge as true $\mathrm{SRH}$, unlike $\mathrm{AB}$, because finding NBVV and AC hidden under accumulated massive clots in the lumen filled with blood, is tremendously difficult. Yet, gel injection can wash out massive blood clots, and as shown in Fig. 1B, AC of a diverticulum can be detected easily. The gel immersion method was first reported in Japan by Yano et al. This method was used for 17 GI patients, and 3 among these, had right-sided CDB. ${ }^{22}$ Because they did not consider the efficacy of OS-1 Jelly in detecting small NBVV and AC in CDB, we mainly focused on the effectiveness of the gel immersion method in patients with massive right-sided diverticular bleeding. Although the num- ber of patients was limited, and the study was retrospective and was performed at a single center without a control group, we showed that OS-1 Jelly injection is a useful technique for difficult situations. Oguri et al. reported the relatively equal distribution of SRH as follows: AB, 34.9\%; NBVV, 32.5\%; and $\mathrm{AC}, 32.5 \%$; this meant that $65 \%$ of stigmata were NBVV and AC. ${ }^{12}$ Thus, improvement of NBVV and AC detection rate should be considered as the key for CDB management. By applying the gel immersion method, we considered that the detection of SRH would improve and the rate of re-bleeding and readmission would decrease. We consider the gel immersion method to be efficient for patients with right-sided CDB, increasing the detection rate of not only $\mathrm{AB}$ but also NBVV and AC.

Conflicts of Interest

The authors have no potential conflicts of interest.

Funding

None.

ORCID

Kazuki Yamamoto:

Yasutoshi Shiratori:

https://orcid.org/0000-0001-6614-2763

https://orcid.org/0000-0002-7784-6210

Takashi Ikeya:

\section{Supplementary Material}

Video 1. An example of the utility of the gel immersion method for the detection of the non-bleeding visible vessel in the presence of massive bleeding (https://doi.org/10.5946/ce.2020.081.v001).

\section{REFERENCES}

1. Strate LL, Gralnek IM. ACG clinical guideline: management of patients with acute lower gastrointestinal bleeding. Am J Gastroenterol 2016;111:459-474.

2. Ghassemi KA, Jensen DM. Lower GI bleeding: epidemiology and management. Curr Gastroenterol Rep 2013;15:333.

3. Gralnek IM, Neeman Z, Strate LL. Acute lower gastrointestinal bleeding. N Engl J Med 2017;376:1054-1063.

4. Painter NS, Burkitt DP. Diverticular disease of the colon, a 20th century problem. Clin Gastroenterol 1975;4:3-21.

5. Warner E, Crighton EJ, Moineddin R, Mamdani M, Upshur R. Fourteen-year study of hospital admissions for diverticular disease in Ontario. Can J Gastroenterol 2007;21:97-99.

6. Nagata N, Niikura R, Aoki T, et al. Increase in colonic diverticulosis and diverticular hemorrhage in an aging society: lessons from a 9-year colonoscopic study of 28,192 patients in Japan. Int J Colorectal Dis 2014;29:379-385.

7. Imaeda H, Hibi T. The burden of diverticular disease and its complications: West versus East. Inflamm Intest Dis 2018;3:61-68.

8. Sugihara K, Muto T, Morioka Y, Asano A, Yamamoto T. Diverticular disease of the colon in Japan. A review of 615 cases. Dis Colon Rectum 1984;27:531-537.

9. Markham NI, Li AK. Diverticulitis of the right colon--experience from Hong Kong. Gut 1992;33:547-549. 
10. Ngoi SS, Chia J, Goh MY, Sim E, Rauff A. Surgical management of right colon diverticulitis. Dis Colon Rectum 1992;35:799-802.

11. Niikura R, Nagata N, Aoki T, et al. Predictors for identification of stigmata of recent hemorrhage on colonic diverticula in lower gastrointestinal bleeding. J Clin Gastroenterol 2015;49:e24-e30.

12. Oguri N, Ikeya T, Kobayashi D, et al. Effectiveness of risk scoring systems in predicting endoscopic treatment in colonic diverticular bleeding. J Gastroenterol Hepatol 2020;35:815-820.

13. McGuire HH Jr. Bleeding colonic diverticula. A reappraisal of natural history and management. Ann Surg 1994;220:653-656.

14. Longstreth GF. Epidemiology and outcome of patients hospitalized with acute lower gastrointestinal hemorrhage: a population-based study. Am J Gastroenterol 1997;92:419-424.

15. Jensen DM, Ohning GV, Kovacs TO, et al. Natural history of definitive diverticular hemorrhage based on stigmata of recent hemorrhage and colonoscopic Doppler blood flow monitoring for risk stratification and definitive hemostasis. Gastrointest Endosc 2016;83:416-423.

16. Nakatsu S, Yasuda H, Maehata T, et al. Urgent computed tomography for determining the optimal timing of colonoscopy in patients with acute lower gastrointestinal bleeding. Intern Med 2015;54:553-558.

17. Mohammed Ilyas MI, Szilagy EJ. Management of diverticular bleeding: evaluation, stabilization, intervention, and recurrence of bleeding and indications for resection after control of bleeding. Clin Colon Rectal
Surg 2018;31:243-250.

18. Jensen DM, Machicado GA, Jutabha R, Kovacs TO. Urgent colonoscopy for the diagnosis and treatment of severe diverticular hemorrhage. $\mathrm{N}$ Engl J Med 2000;342:78-82.

19. Gilshtein H, Kluger Y, Khoury A, Issa N, Khoury W. Massive and recurrent diverticular hemorrhage, risk factors and treatment. Int J Surg 2016;33 Pt A:136-139.

20. Faucheron JL, Roblin X, Bichard P, Heluwaert F. The prevalence of right-sided colonic diverticulosis and diverticular haemorrhage. Colorectal Dis 2013;15:e266-e270.

21. Ishii N, Itoh T, Uemura M, et al. Endoscopic band ligation with a water-jet scope for the treatment of colonic diverticular hemorrhage. Dig Endosc 2010;22:232-235.

22. Yano T, Nemoto D, Ono K, et al. Gel immersion endoscopy: a novel method to secure the visual field during endoscopy in bleeding patients (with videos). Gastrointest Endosc 2016;83:809-811.

23. East JE, Suzuki N, Stavrinidis M, Guenther T, Thomas HJ, Saunders BP. Narrow band imaging for colonoscopic surveillance in hereditary non-polyposis colorectal cancer. Gut 2008;57:65-70.

24. Obana T, Fujita N, Sugita R, et al. Prospective evaluation of contrast-enhanced computed tomography for the detection of colonic diverticular bleeding. Dig Dis Sci 2013;58:1985-1990. 